\title{
Accuracy and Safety of Percutaneous Lumbosacral Pedicle Screw Placement Using Dual-Planar Intraoperative Fluoroscopy
}

\author{
Conor Dunn ${ }^{1}$, Michael Faloon ${ }^{1}$, Edward Milman ${ }^{1}$, \\ Sina Pourtaheri ${ }^{2}$, Kumar Sinah ${ }^{1}$, Ki Hwang ${ }^{1}$, Arash Emami ${ }^{1}$ \\ ${ }^{1}$ Seton Hall University School of Health and Medical Science, Wayne, NJ, USA \\ ${ }^{2}$ Department of Orthopaedic Surgery, University of California Los Angeles, Los Angeles, CA, USA
}

Study Design: Retrospective case series with prospective arm.

Purpose: To assess the safety and accuracy of percutaneous lumbosacral pedicle screw placement (PLPSP) in the lumbosacral spine using intraoperative dual-planar fluoroscopy (DPF).

Overview of Literature: There are several techniques available for achieving consistent, safe, and accurate results with PLPSP. There is a paucity of literature describing the beneficial operative, economic, and clinical outcomes of DPF, the most readily accessible image guidance system.

Methods: From 2004 to 2014, 451 consecutive patients underwent PLPSP using DPF, for a total of 2,345 screw placement. The results of prospectively obtained postoperative computed tomography (CT) examinations of an additional 41 consecutive patients were compared with the results of $104 \mathrm{CT}$ examinations obtained postoperatively due to clinical symptomatology; these results were interpreted by three reviewers. The rates of revision indicated by misplaced screws with consistent clinical symptomatology were compared between groups. Pedicle screw placement was graded according to 2-mm increments in medial pedicle wall breach and measurement of screw axis placement.

Results: Seven of the 2,345 pedicle screws placed percutaneously with the use of the dual-planar fluoroscopic technique required revision because of a symptomatic misplaced screw, for a screw revision rate of $0.3 \%$. There were no statistically significant demographic differences between patients who had screws revised and those who did not. All screws registered greater than $10 \mathrm{~mA}$ on electromyographic stimulation. In the 41 prospectively obtained CT examinations, one out of 141 screws $(0.7 \%)$ was revised due to pedicle wall breach; whereas among the 104 patients with 352 screws, three screws were revised $(0.9 \%)$.

Conclusions: DPF is an extremely accurate, safe, and reproducible technique for placement of percutaneous pedicle screws and is a readily available and cost-effective alternative to CT-guided pedicle screw placement techniques. Postoperative CT evaluation is not necessary with PLPSP unless the patient is symptomatic. Acceptable electromyographic thresholds may need to be reevaluated.

Keywords: Minimally invasive surgical procedures; Lumbosacral region; Pedicle screws; Fluoroscopy

Received Mar 29, 2017; Revised Jun 14, 2017; Accepted Jul 8, 2017

Corresponding author: Conor Dunn

Seton Hall University School of Health and Medical Science, University Spine, 504 Valley Road, Suite 203

Wayne, NJ 07470, USA

Tel: +1-973-686-0700 (x199), Fax: +1-973-686-0701, E-mail: Emamiresearch@gmail.com 


\section{Introduction}

Percutaneous lumbosacral pedicle screw placement (PLP$\mathrm{SP})$, which has a variety of applications and indications, has become a popular and commonly used technique [13]. Despite the popularity of the technique, few large studies have objectively described its safety and accuracy in the clinical setting pertaining to the lumbosacral spine [4]. Though rare, misplaced pedicle screws have been thought to have devastating clinical consequences. Although pedicle screw placement and its efficacy have been thoroughly described, the clinical safety profile of the percutaneous technique has been largely empirical and differs according to the technique and equipment used by the investigating surgeon.

Computed tomography (CT) is considered the gold standard for assessment of pedicle screw placement $[5,6]$. Several trials have utilized CT with two- and threedimensional (2D and $3 \mathrm{D})$ reconstructions to assess the accuracy of screw placement $[6,7]$. Over the past decade, new technologies have provided alternative techniques to improve the accuracy of pedicle screw placement with open and percutaneous techniques, including robotassisted, isocentric C-arm 3D fluoroscopic guidance, computer CT navigational software, electromagneticbased navigation software, and patient-specific templating [8-12]. These imaging guidance systems report accuracy of placement in the high 90th percentiles without pedicle wall breach. However, cost and availability are limiting factors for many hospital systems and investigating surgeons. Compared with other imaging guidance systems, dual fluoroscopy remains a readily available technology that potentially offers decreased cost and intraoperative time.

This study, conducted at a single institution, aimed to describe the safety profile of percutaneous pedicle screw placement systems using standard dual-planar 2D fluoro- scopic imaging in the lumbosacral spine. Secondarily, we aimed to quantify our clinical experience with our radiographic data to formulate an overall accuracy rate of screw placement performed using our technique.

\section{Materials and Methods}

Approval of the Institutional Review Board of St. Joseph's Regional Medical Center was obtained for conducting this study (IRB approval no., PR\#08-034). Analysis of a single institution's database from 2004 to 2014 yielded 451 consecutive cases which were retrospectively reviewed; the patients had undergone underwent anterior, lateral, or minimally invasive transforaminal lumbar interbody fusion (m-TLIF) or traumatic fixation with PLPSP. Percutaneous screws were placed in the lumbosacral spine to provide rigid stabilization support for anterior and lateral interbody fusion. The results from patients with screws that were revised due to persistent clinical symptomatology were compared with the results from patients whose screws were not revised. The groups underwent risk factor analysis, including American Society of Anesthesiologists Physical Status Classification System (ASA grade) grading and recording of complications. Statistical analyses, including calculation of $p$-values, were performed using Student $t$-test for mean values and Fisher's exact test for categorical variables.

All surgical procedures were performed at a single institution. The indications for surgery included degenerative disc disease, post-laminectomy syndrome/recurrent herniated nucleus pulposus, spondylolisthesis, and traumatic fracture and instability. Patients for whom complete medical records and radiographs were available were included in the final analysis.

CT studies of the lumbosacral spine were obtained prospectively from 41 consecutive patients for whom a total of 141 screws were placed. Upon retrospective review of

Table 1. Comparison of retrospectively reviewed and prospectively obtained computed tomography examinations

\begin{tabular}{lcc} 
Variable & Retrospective & Prospective \\
No. of patients & 104 & 41 \\
Men:women ratio & $42: 62$ & $18: 23$ \\
No. of screws placed & 352 & 141 \\
No. of screws revised (\%) & $3(0.9)$ & $1(0.7)$ \\
\hline No. of screws confirmed in pedicle (\%) & $326(92.6)$ & $133(95.0)$ \\
\hline
\end{tabular}


the medical records and imaging studies of 178 consecutive patients, CT images were obtained for review from 104 patients (352 screws). The rates of revision, indicated by a confirmed misplaced screw, with corresponding clinical symptomatology (Table 1), as well as CT evaluation of screw placement according to level (Fig. 1), were compared between prospective and retrospective groups. The prospective group was then discontinued as there was no statistically significant difference between the groups in the rate of revision $(0.7 \%$ in the prospective group and $0.9 \%$ in the retrospective group, $p=1.000$ ), which was indicated by a confirmed misplaced screw with corresponding clinical symptomatology.

All CT examinations (41 in the prospective group, 104 in the retrospective group) were evaluated independently by two fellowship-trained orthopedic spine surgeons and a fellowship-trained neuroradiologist. Each reviewer graded pedicle screw placement according to three criteria: dichotomous placement within the cortical confines of pedicle walls, wall breach increments $(<2 \mathrm{~mm}$ or $<4 \mathrm{~mm})$, and the placement of pedicle screw central axis within the cortical confines of the pedicle wall. Statistical analysis was performed using Fleiss's unweighted $\kappa$-coefficient to describe interobserver reliability. The reviewers were blinded to each other's results. The results of the review and analysis (according to level) are shown in Fig. 1.

For the surgery, the patient was placed in the prone position on a radiolucent Jackson frame. One or two fluoroscopic $\mathrm{C}$-arm imaging machines were utilized under direct anterior-posterior $(\mathrm{A} / \mathrm{P})$ images of the pedicles and positioned in the direct lateral image (Fig. 2). The approximate screw trajectory was estimated with an 18-gauge spinal needle. A stab incision 1 to $2 \mathrm{~cm}$ in length was made in the skin. A Jamshidi needle was then placed transmuscularly to a starting point at the junction of the transverse process and the lateral border of the facet joint. Confirmation of needle placement at the lateral wall of the pedicle was obtained with the $\mathrm{A} / \mathrm{P}$ view, and the needle was advanced to the medial pedicle wall, and its trajectory into the vertebral body was seen on the lateral projection.

Table 2. Patient demographics overall and compared between cohorts

\begin{tabular}{|c|c|c|c|c|}
\hline Demographic & Overall & $\begin{array}{l}\text { No pedicle wall } \\
\text { breach cohort }\end{array}$ & $\begin{array}{l}\text { Revision, pedicle wall } \\
\text { breach cohort }\end{array}$ & $p$-value \\
\hline No. of patients & 451 & 444 (98.5) & $7(1.6)$ & \\
\hline Screws & 2,345 & 2,338 (99.7) & $7(0.3)$ & \\
\hline Age (yr) & $56.0(23-76)$ & $55.9(23-76)$ & $57.3(43-68)$ & 0.6045 \\
\hline Men:women ratio & $194: 257$ & $191: 253$ & $3: 4$ & 1.0000 \\
\hline Body mass index $\left(\mathrm{kg} / \mathrm{m}^{2}\right)$ & 28.6 & 28.5 & 29.2 & 0.7807 \\
\hline ASA grade & $1.9(1-3)$ & $1.9(1-3)$ & $2(1-3)$ & 0.5449 \\
\hline Smoking history & $39(8.7)$ & $38(9)$ & $1(14.3)$ & 0.4425 \\
\hline \multicolumn{5}{|l|}{ Primary operative indication } \\
\hline Degenerative disc disease & $190(42.1)$ & $187(42.1)$ & 3 & 1.0000 \\
\hline Spondylolisthesis & $68(15.1)$ & $67(15.1)$ & 1 & 1.0000 \\
\hline Post-laminectomy syndrome/recurrent herniated disc & $161(35.7)$ & $158(35.6)$ & 3 & 0.7041 \\
\hline Traumatic fracture/instability & $32(7.1)$ & $32(7.2)$ & 0 & 1.0000 \\
\hline \multicolumn{5}{|l|}{ Surgery } \\
\hline Anterior LIF & $195(43.2)$ & $192(43.2)$ & 3 & 1.0000 \\
\hline Minimally invasive transforaminal LIF & $164(36.4)$ & $161(36.2)$ & 3 & 0.7086 \\
\hline Lateral LIF & $60(13.3)$ & $59(13.2)$ & 1 & 1.0000 \\
\hline Traumatic fixation & $32(7.1)$ & $32(7.2)$ & 0 & 1.0000 \\
\hline
\end{tabular}

Values are presented as number (\%) or mean (range), unless otherwise stated.

LIF, lumbar interbody fusion.

a)American Society of Anesthesiologists Physical Status Classification System grade. 


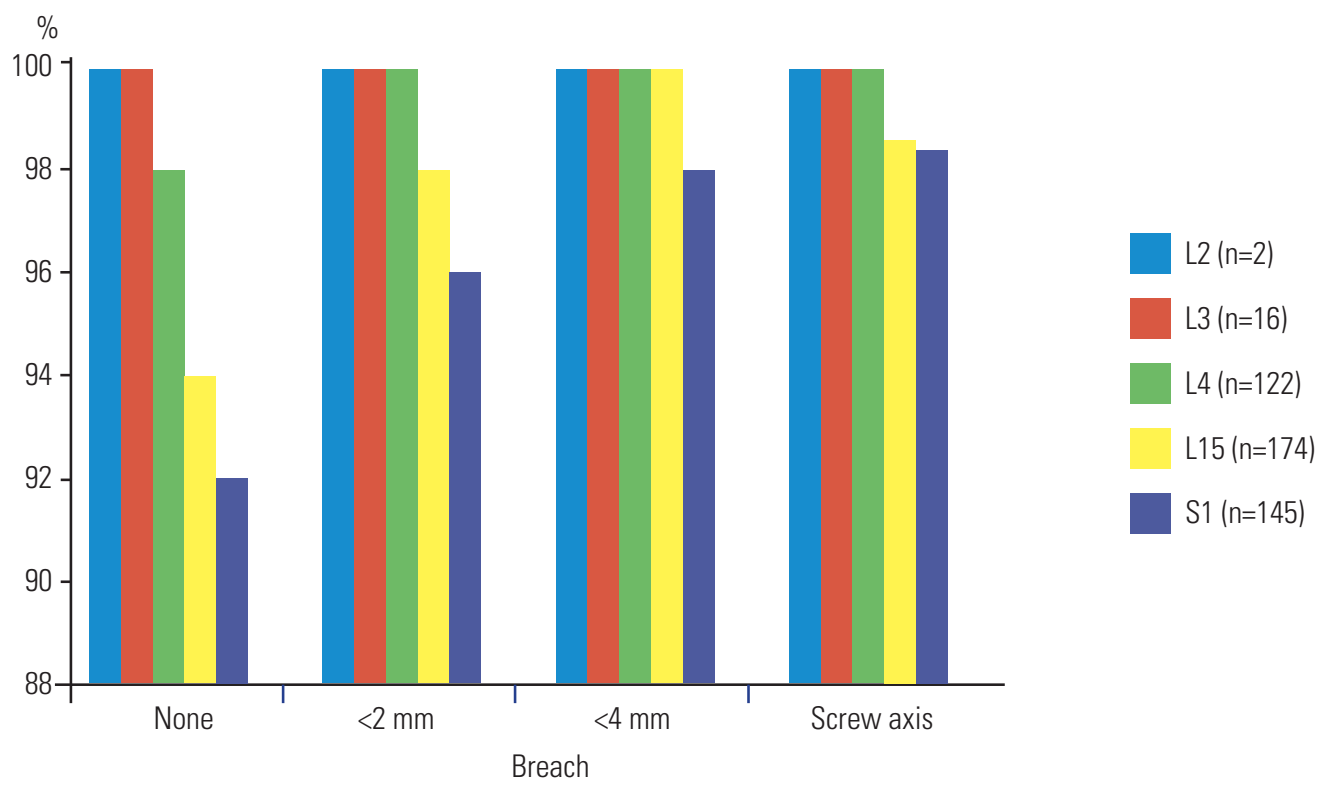

Fig. 1. Computed tomography evaluation of accuracy of screw placement according to level.

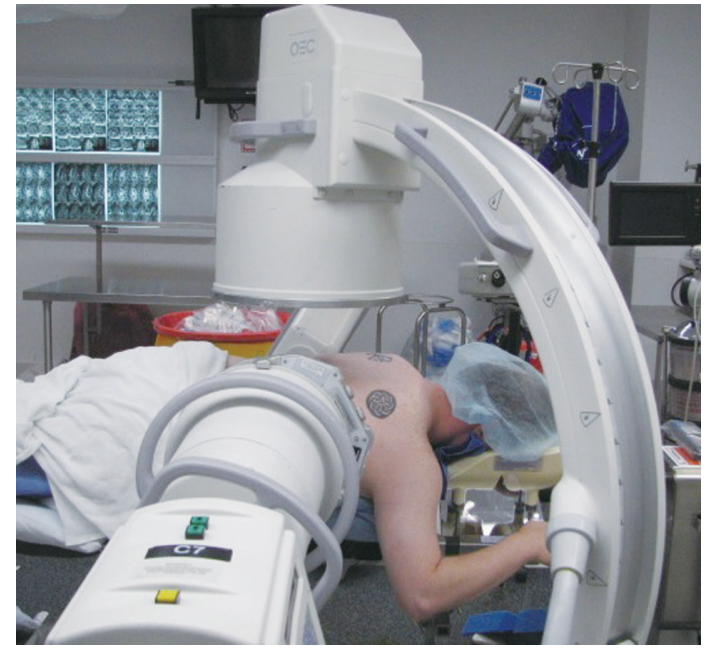

Fig. 2. Two fluoroscopic C-arm machines in the anterior/posterior and lateral positions.

The needle was incrementally advanced down the pedicle, and serial fluoroscopic images confirmed placement within the pedicle walls. Once placement within the vertebral body was confirmed on both images, a guidewire was introduced through the cannulated trocar, which was then removed.

The incision was then dilated with a series of tubes for introduction of pedicle preparation tools. Over the guidewire, the cortex was prepared with an awl and the pedicle was tapped. Proper placement of the tap was confirmed by fluoroscopic imaging. The tap was then tested to ensure that electromyographic (EMG) thresholds had been adequately maintained. The appropriately measured cannulated screw was then passed over the guidewire into the desired location. The placement was once again verified by dual imaging. The tap and the screw were then checked individually with electrically elicited, triggered EMG for possible pedicle wall breach. The intersegmental rods were then passed percutaneously through the most proximal incision, and each screw was capped through the respective incision site. Final images confirmed the placement of the instrumentation.

\section{Results}

A total of 451 consecutive patients had PLPSP placed in the lumbosacral region with the use of the dual-planar fluoroscopic placement technique. Complete medical records, operative reports, clinical notes, and radiographs were available for all 451 patients (2,345 screws). The demographic characteristics of patients who had a screw revised after suffering postoperative radiculopathy secondary to pedicle wall breach were compared with those of patients who did not have a screw revised.

In total, there were 257 women and 194 men aged between 23 and 76 years, with a mean age of 56 (standard deviation $=8.4$ ) years. Of these, 164 patients underwent augmentation with m-TLIF (36.4\%), 195 with anterior 
lumbar interbody fusion (ALIF, 43.2\%), 60 with lateral lumbar interbody fusion (LLIF, 13.3\%), and 32 with internal fixation of traumatic instability (7.1\%), for a total of 2,345 screws. Table 2 shows the demographic characteristics of the overall population, of those who underwent revision due to symptomatic pedicle wall breach of screws, and of those who did not undergo revision. There were no differences between the cohorts in terms of sex ratio, operative indication, type of surgery, body mass index, ASA grade, and mean age at the time of surgery.

The overall rate of revisions for patients who returned to the operating room for any complication was $7.5 \%(n=34)$. Seven screws were revised in seven patients $(1.6 \%)$, for a $0.3 \%$ revision rate per screw placed; one patient returned to the operating room after rod disengagement; 20 patients underwent revision surgery for symptomatic adjacent segment degeneration; two patients underwent revision surgery for instrumentation failure or hardware prominence; one patient had a surgical site infection that was treated with surgical washout and intravenous antibiotics; one patient had a headache due to incidental durotomy, which self-resolved; and one patient had a postoperative hematoma. All patients achieved good functional and clinical outcomes.

Table 1 shows the results of statistical analysis and comparison between patients of the prospective and retrospective groups. They were compared according to the rate of revision surgery, indicated by a confirmed misplaced screw with corresponding clinical symptomatology. In the 41 consecutive patients with prospectively obtained CT examinations, a total of 141 screws were placed, with only one revision $(0.7 \%)$ indicated by a confirmed misplaced screw with corresponding clinical symptomatology. In the 104 patients for whom CT images were available for review in the retrospective group, three screws required revision $(0.9 \%)$, indicated by a confirmed misplaced screw with corresponding clinical symptomatology. The prospective group was then discontinued, as there was no statistically significant difference between the groups in the rate of revision.

Fig. 1 shows the accuracy of placement according to level. No statistically significant differences were found between levels in accuracy rate. Two screws with axis breach were placed in the $\mathrm{S} 1$ pedicle (one on the right side and the other on the left), and another was placed on the left side of L5.

\section{Discussion}

The utility of pedicle screws has been well described for a variety of surgical indications [7,13-15]. Numerous techniques and approaches for placement of pedicle screws in the lumbosacral spine have also been described. Although Magerl [16] described percutaneous placement of pedicle screws for the purpose of securing an external spinal fixator for the treatment of spinal instability in the setting of acute trauma or osteomyelitis in 1977, systems have been developed for the broad employment of their usage only recently. There are a variety of factors, such as demonstrated accuracy, cost, availability, exposure of the patient and personnel to radiation, setup time, and intraoperative time, which are unique to each technique; these factors must be weighed by the surgeon before deciding on the most appropriate choice for a patient. Utilizing dualplanar fluoroscopic imaging simultaneously in the lateral and $\mathrm{A} / \mathrm{P}$ positions is one option that we describe here as safe, accurate, readily available, requiring minimal setup time and possibly cost-effective, with less exposure of the patient, surgeon, and staff to radiation than in intraoperative CT.

Although the popularity of percutaneous placement systems has risen, clinical safety data describing placement in the lumbar spine have come largely from pilot studies and are sparse, partially because of variation in techniques and implementation systems. Patients in this data set underwent ALIF, LLIF, m-TLIF, or fixation of traumatic instability with the use of dual-planar fluoroscopic visualization with adequate EMG threshold confirmation. To our knowledge, our study is the largest systematic clinical analysis at a single institution of percutaneously placed lumbosacral pedicle screws utilizing dual-planar fluoroscopy, the most readily available technique.

Historically, the primary safety concerns with pedicle screw placement have been complications related to rare neurologic or vascular injuries [15]. Anteriorly misplaced screws can have potentially severe complications due to penetration of vascular, visceral, ureteral, sympathetic, and neural structures by the screws [17]. Neurological injury is suspected in association with medial or inferior pedicle wall breach, although the severity of such injuries has not been clearly demonstrated $[3,14,17]$. No significant complications related to screw malpositioning, other than persistent radiculopathy, were observed in this series.

Today, CT is regarded as the "gold standard" for evalu- 
ating the accuracy of pedicle screw placement, revealing 10 times more medial wall perforations than standard radiographs. However, the criteria for accuracy of measurements vary in the literature, ranging from encroachment of the pedicle wall to millimeters of breach [2,10,18-20]. A break $>6 \mathrm{~mm}$ suggests a high likelihood of correlated neurologic injury $[7,13]$. Despite variation in the accuracy of placement due to differences in grading criteria, metaanalyses in 2007 and 2015 found accuracy rates of $91.3 \%$ and $91.4 \%$, respectively $[2,4]$. Overall, our measured accuracy rate of $93.1 \%$ of screws confirmed by CT scan without breach falls within the range of previously published data and validates the efficacy of utilizing dual-planar fluoroscopy in conjunction with EMG thresholds [21,22].

Central screw axis has previously been described as a grading criterion in the lumbosacral spine in order to determine a clinically relevant grading system [23]. In our initial CT-driven series, 493 pedicle screws were placed. Our study design aimed to detect any differences in the accuracy of screw placement between a retrospective group of patients who had had CT imaging studies performed to detect sources of persistent postoperative pain and a prospective group of patients with CT images obtained as part of routine protocol. Our results indicated no significant differences between the groups in the accuracy rates according to each of the grading criteria. Four patients had radicular symptoms postoperatively that were demonstrated to correlate with CT images demonstrating medial pedicle wall breach. These four patients had complete screw axis breach of the medial pedicle wall, and all complained of radicular symptoms that anatomically correlated with postoperative CT imaging. Each of these patients had $>4 \mathrm{~mm}$ of medial pedicle wall breach. None of the remaining screws with medial wall breach $<4 \mathrm{~mm}$ were placed in patients who had radicular symptoms that were anatomically correlated with instrument placement. Thus, $0.8 \%$ of screws placed had radiographic images that were shown to cause clinical symptoms. No screws were associated with clinically significant vascular injury.

Additionally, our purely retrospective series included 2,304 screws, of which only six were revised due to symptomatic medial pedicle wall breach, for a screw accuracy rate of $99.7 \%$. It should be noted that three of these screws were revised during the first 3 years of the study and the remaining three were revised during the final 7 years, which supports the technical learning curve hypothesis previously described in the literature $[6,20,24]$. This level of accuracy rivals that obtained with the newest and most advanced navigational software, robotic-assisted instrumentation, and patient-specific templating. The system remains readily available to most surgeons in the community, has minimal preoperative setup time, is costeffective, and has reduced radiation exposure compared with intraoperative CT-guided three-dimensional systems $[9,12,25,26]$. A recent study done at a level 1 trauma center directly compared more than 2,000 pedicle screws placed with the use of a conventional open technique with single fluoroscopic guidance or placed with a computernavigated placement system and found no increase in accuracy or safety in the computer-navigated group [27].

Analysis of patients examined by CT according to the level of screw placement found no statistically significant differences between levels in placement accuracy rates. Of the four screws in the initial study that correlated with radicular pain, two were placed at the S1 level and two at the L5 level; three of the patients were in the retrospectively analyzed group and the other patient was in the prospectively analyzed group. In our sampling, there was a large predilection for the L4, L5, and S1 levels, which had a strong correlation with our population's primary diagnoses. In a study in 2014 by Smith et al. [28], both symptomatic pedicle wall breaches were found in the L5 segment, whereas L3 had the highest overall rate of breach. In 2015, Chiu et al. [29] showed that greater caution must be taken during the insertion of L5 and S1 percutaneous pedicle screws because of the more angulated pedicles of these segments, the anatomical variations in their vertebral bodies, and the morphology of the spinal canal at this location. Finally, the patient's body habitus and excessive posterior lumbar subcutaneous adipose tissue greatly affect the placement angle. A limitation of the present study of technical concerns regarding placement and EMG values was the patients' obesity status.

EMG threshold values for acceptable screw placement have been established for open pedicle screw placement $[11,30]$. Proper technique and interpretation of results allow for early repositioning and verification of the correct trajectory. No standardized threshold protocol has been established for PLPSP due to the disbursement of current via conductive soft tissues secondary to the use of insulation instrumentation [21]. Part of our screw placement protocol included the placement of both the (metal) tap and each screw itself. The two screws revised from the initial study were noted to have values acceptable $(<12$ 
$\mathrm{mA}$ ) for the standard open techniques; however, both patients were symptomatic, and CT scan revealed screw axis breach of the medial pedicle wall. Both patients had improved symptoms after revision of screw placement. This suggests that perhaps the EMG threshold criteria for percutaneous placement should be reevaluated. In 2006, Ozgur et al. [22] reported using a threshold of $10 \mathrm{~mA}$ to dictate repositioning, with satisfactory results.

To the best of our knowledge, this study is the largest systematic clinical analysis of percutaneously placed lumbosacral pedicle screws utilizing this readily available technique. Although empirical data can be extrapolated from previous studies to support the safety of this technique, our study quantifies the clinical safety profile in one institution's experience. It also adds to the argument that screw axis breach is associated with a significant correlation between radiographic imaging and patient symptoms. Finally, our findings raise the question of the application of EMG thresholds used for open placement of screws and whether or not these values are applicable to percutaneous techniques.

\section{Conclusions}

PLPSP using intraoperative dual-planar fluoroscopy is a safe technique with low rates of complication and revision. Routine postoperative CT is not necessary to ensure safe screw placement in asymptomatic patients. With percutaneously measured EMG potentials, screw placement may not correlate with previously described thresholds.

\section{Conflict of Interest}

No potential conflict of interest relevant to this article was reported.

\section{References}

1. Scheufler KM, Dohmen H, Vougioukas VI. Percutaneous transforaminal lumbar interbody fusion for the treatment of degenerative lumbar instability. Neurosurgery 2007;60(4 Suppl 2):203-12.

2. Kosmopoulos V, Schizas C. Pedicle screw placement accuracy: a meta-analysis. Spine (Phila Pa 1976) 2007;32:E111-20.

3. Foley KT, Gupta SK. Percutaneous pedicle screw fixation of the lumbar spine: preliminary clinical results.
J Neurosurg 2002;97(1 Suppl):7-12.

4. Aoude AA, Fortin M, Figueiredo R, Jarzem P, Ouellet J, Weber MH. Methods to determine pedicle screw placement accuracy in spine surgery: a systematic review. Eur Spine J 2015;24:990-1004.

5. Chapman TM, Blizzard DJ, Brown CR. CT accuracy of percutaneous versus open pedicle screw techniques: a series of 1609 screws. Eur Spine J 2016;25:1781-6.

6. Bydon M, Xu R, Amin AG, et al. Safety and efficacy of pedicle screw placement using intraoperative computed tomography: consecutive series of 1148 pedicle screws. J Neurosurg Spine 2014;21:320-8.

7. Castro WH, Halm H, Jerosch J, Malms J, Steinbeck J, Blasius S. Accuracy of pedicle screw placement in lumbar vertebrae. Spine (Phila Pa 1976) 1996;21:1320-4.

8. Farshad M, Betz M, Farshad-Amacker NA, Moser M. Accuracy of patient-specific template-guided vs. freehand fluoroscopically controlled pedicle screw placement in the thoracic and lumbar spine: a randomized cadaveric study. Eur Spine J 2017;26:738-49.

9. Nakashima H, Sato K, Ando T, Inoh H, Nakamura $\mathrm{H}$. Comparison of the percutaneous screw placement precision of isocentric C-arm 3-dimensional fluoroscopy-navigated pedicle screw implantation and conventional fluoroscopy method with minimally invasive surgery. J Spinal Disord Tech 2009;22:468-72.

10. Laine T, Lund T, Ylikoski M, Lohikoski J, Schlenzka D. Accuracy of pedicle screw insertion with and without computer assistance: a randomised controlled clinical study in 100 consecutive patients. Eur Spine J 2000;9:235-40.

11. Glassman SD, Dimar JR, Puno RM, Johnson JR, Shields $\mathrm{CB}$, Linden $\mathrm{RD}$. A prospective analysis of intraoperative electromyographic monitoring of pedicle screw placement with computed tomographic scan confirmation. Spine (Phila Pa 1976) 1995;20:1375-9.

12. Marcus HJ, Cundy TP, Nandi D, Yang GZ, Darzi A. Robot-assisted and fluoroscopy-guided pedicle screw placement: a systematic review. Eur Spine J 2014;23:291-7.

13. Schulze CJ, Munzinger E, Weber U. Clinical relevance of accuracy of pedicle screw placement: a computed tomographic-supported analysis. Spine (Phila Pa 1976) 1998;23):2215-20.

14. Weinstein JN, Rydevik BL, Rauschning W. Anatomic 
and technical considerations of pedicle screw fixation. Clin Orthop Relat Res 1992;(284):34-46.

15. Lehman RA Jr, Lenke LG, Keeler KA, Kim YJ, Cheh G. Computed tomography evaluation of pedicle screws placed in the pediatric deformed spine over an 8-year period. Spine (Phila Pa 1976) 2007;32:2679-84.

16. Magerl FP. Stabilization of the lower thoracic and lumbar spine with external skeletal fixation. Clin Orthop Relat Res 1984;(189):125-41.

17. Whitecloud TS, Skalley TC, Cook SD, Morgan EL. Roentgenographic measurement of pedicle screw penetration. Clin Orthop Relat Res 1989;(245):57-68.

18. Weinstein JN, Spratt KF, Spengler D, Brick C, Reid S. Spinal pedicle fixation: reliability and validity of roentgenogram-based assessment and surgical factors on successful screw placement. Spine (Phila Pa 1976) $1988 ; 13: 1012-8$.

19. Kim YJ, Lenke LG. Thoracic pedicle screw placement: free-hand technique. Neurol India 2005;53:512-9.

20. Gertzbein SD, Robbins SE. Accuracy of pedicular screw placement in vivo. Spine (Phila Pa 1976) 1990;15:11-4.

21. Wang MY, Pineiro G, Mummaneni PV. Stimulusevoked electromyography testing of percutaneous pedicle screws for the detection of pedicle breaches: a clinical study of 409 screws in 93 patients. J Neurosurg Spine 2010;13:600-5.

22. Ozgur BM, Berta S, Khiatani V, Taylor WR. Automated intraoperative EMG testing during percutaneous pedicle screw placement. Spine J 2006;6:708-13.

23. Kim YJ, Lenke LG, Cheh G, Riew KD. Evaluation of pedicle screw placement in the deformed spine using intraoperative plain radiographs: a comparison with computerized tomography. Spine (Phila Pa 1976) 2005;30:2084-8.
24. Von Jako R, Finn MA, Yonemura KS, et al. Minimally invasive percutaneous transpedicular screw fixation: increased accuracy and reduced radiation exposure by means of a novel electromagnetic navigation system. Acta Neurochir (Wien) 2011;153:589-96.

25. Ito Y, Sugimoto Y, Tomioka M, Hasegawa Y, Nakago K, Yagata Y. Clinical accuracy of 3D fluoroscopyassisted cervical pedicle screw insertion. J Neurosurg Spine 2008;9:450-3.

26. Slomczykowski M, Roberto M, Schneeberger P, Ozdoba C, Vock P. Radiation dose for pedicle screw insertion: fluoroscopic method versus computerassisted surgery. Spine (Phila Pa 1976) 1999;24:97582.

27. Kraus M, Weiskopf J, Dreyhaupt J, Krischak G, Gebhard F. Computer-aided surgery does not increase the accuracy of dorsal pedicle screw placement in the thoracic and lumbar spine: a retrospective analysis of 2,003 pedicle screws in a level I trauma center. Global Spine J 2015;5:93-101.

28. Smith ZA, Sugimoto K, Lawton CD, Fessler RG. Incidence of lumbar spine pedicle breach after percutaneous screw fixation: a radiographic evaluation of 601 screws in 151 patients. J Spinal Disord Tech 2014;27:358-63.

29. Chiu CK, Kwan MK, Chan CY, Schaefer C, HansenAlgenstaedt N. The accuracy and safety of fluoroscopically guided percutaneous pedicle screws in the lumbosacral junction and the lumbar spine: a review of 880 screws. Bone Joint J 2015;97-B:1111-7.

30. Raynor BL, Lenke LG, Bridwell KH, Taylor BA, Padberg AM. Correlation between low triggered electromyographic thresholds and lumbar pedicle screw malposition: analysis of 4857 screws. Spine (Phila Pa 1976) 2007;32:2673-8. 